\title{
Phospholipid bilayers in model membranes and drug delivery systems: from physics to pharmacy
}

Special Issue Article

Uhríková D.凶

Department of Physical Chemistry of Drugs, Faculty

of Pharmacy, Comenius University in Bratislava

Odbojárov 10, 83232 Bratislava, Slovakia

Received 16 June, 2021, accepted 17 June, 2021

Abstract Lipids spontaneously aggregate in an aqueous environment forming supramolecular structures of various architectures known as liquid crystalline mesophases. Their thermodynamic properties determined by dual polar/apolar nature coupled with the possibility to modulate the structural parameters, phase geometry and stability are challenging for applications in drug delivery systems. We review a few examples of functionality of lipid bilayers.

Keywords lipid bilayer-non-lamellar phases-antimicrobial peptides-lipoplexes-pulmonary surfactant

Amphiphilic molecules of lipids self-assemble in water to minimise the exposure of their hydrophobic moieties to water. It was found that both the energetics at the lipid-water interface and the lipid molecular shape play a very important role in the aggregation and formation of resultant structures. While the hydrophobic interaction has a tendency to decrease the total surface area, repulsive interactions tend to increase the surface area. These opposing forces give rise to an optimal equilibrium area per lipid molecule. Packing restrictions determine the curvature of lipid monolayer and can also give rise to an optimum aggregate size (Israelachvili et al., 1976). Thus, the hydrophobic effect and structural diversity of lipidic molecules are responsible for the formation of a high variety of their supra-molecular assemblies. Fig. 1 illustrates a few structures of the lyotropic liquid crystalline mesophases formed by lipids: (A) one-dimensional lamellar phase known as multilamellar liposomes (onion-like structure) in excess of water; (B) two-dimensional columnar hexagonal phase and $(C)$ three-dimensional cubic phases of symmetries characterised by space groups. Multilamellar and particularly unilamellar liposomes formed by single lipid bilayer frequently serve as a model system of lipid bilayer of biological membrane. Lipidic mesophases attract attention due to their capability to accommodate a drug into both the water phase and the hydrophobic matrix. Since 1975, when the first demonstration of the improved in vivo activity of liposome-entrapped anticancer drug in animal models proved successful treatment of mice bearing leukaemia (Kobayashi et al., 1975), a wide variety of lipidic drug delivery systems have been employed. Here, we review a few examples of functionality of lipid bilayers.

\section{LIPID BILAYERS AS A MODEL OF BIOLOGICAL MEMBRANE}

Antimicrobial peptides (AMPs) emerged as an interesting alternative to antibiotics that fight against infectious diseases. AMPs are considered membrane-active agents leading to cell death by acting on the phospholipid membrane. All proposed bactericidal mechanisms have the same main initialisation adsorption of AMPs onto the membrane due to electrostatic interactions between the cationic peptides and the accessible anionic groups of hydrophilic phospholipid headgroups at the membrane surface. Thereafter, accumulation and positional change eventually lead to the formation of pores, membrane permeabilisation or its micellisation (Teixeira et al., 2012). The interaction must be selective regarding the distinction between mammalian cells and pathogen cells (bacteria, fungi, protozoa). Thus, knowledge of the details of AMPs interaction with lipid bilayers of composition mimicking the pathogen membrane is of great importance to evaluate their antimicrobial activity. Small-angle X-ray and neutron scattering (SAXS and SANS, respectively) studies can 

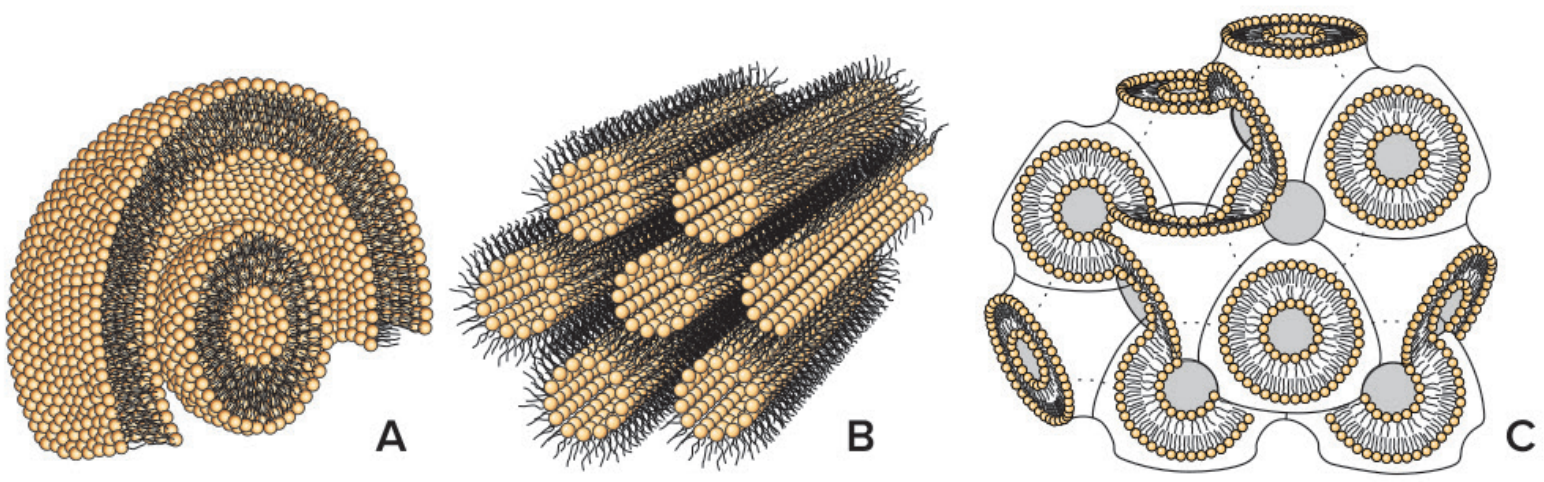

Figure 1. Lyotropic liquid crystalline mesophases: $(A)$ multilamellar vesicles; $(B)$ inverted hexagonal phase; (C) cubic phase of Pn3m space group.

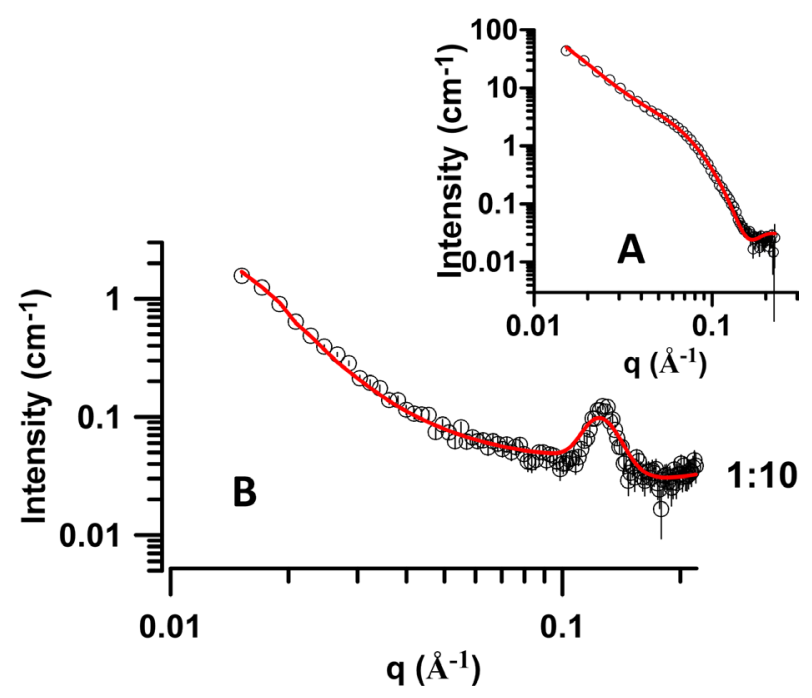

Figure 2. (A) SANS curves of POPE/POPG vesicles; (B) CAM$P O P E / P O P G$ at $P: L=1: 10 \mathrm{~mol} / \mathrm{mol}(P O P E / P O P G=3: 1 \mathrm{~mol} / \mathrm{mol})$ Full lines represent fitting curves.

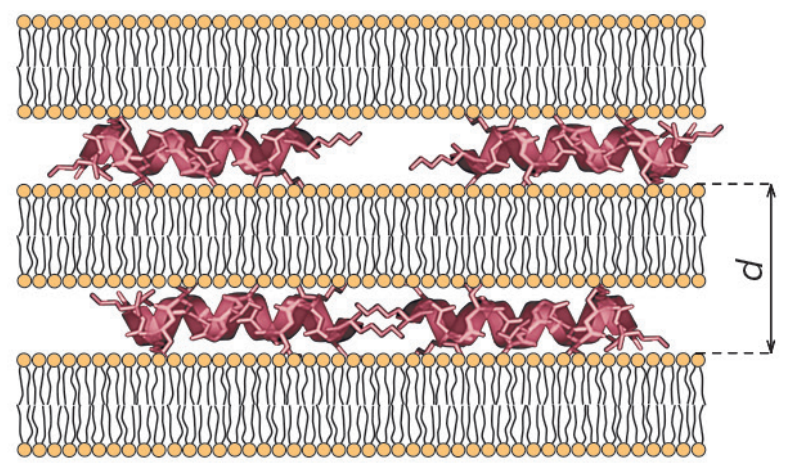

Figure 3. Multilamellar CAM-POPE/POPG structure identified at liquid crystalline state of the lipids $\left(36^{\circ} \mathrm{C}\right) . d$-repeat distance. give the necessary information on the AMPs' ability to affect the structure and integrity of the membrane.

We studied the interaction of cecropin A-melittin (CAM, nominal charge +6), a hybrid peptide composed of the cationic region of cecropin $A$ and the hydrophobic and non-haemolytic region of melittin with a bacterial model lipid membrane composed of zwitterionic palmitoyl-oleoylglycerophosphoethanolamine (POPE) and negatively charged palmitoyl-oleoyl-phosphoglycerol (POPG). SANS and SAXS were used to unravel the mechanism of the peptide antimicrobial activity as described in Silva et al. (2018). Fig. 2A depicts the normalised SANS intensity as a function of the scattering vector $q$ of POPE/POPG oligolamellar vesicles with the lipid bilayer thickness being $d_{L} \sim 39.4 \AA$. SANS data were analysed using SasView software. Fig. $2 B$ shows the $I(q)$ of CAM-POPE/POPG mixtures at P:L $=1: 10 \mathrm{~mol} / \mathrm{mol}$ and $36^{\circ} \mathrm{C}$. When the vesicle dispersion is mixed with the CAM peptide, a fine white precipitate spontaneously forms, suggesting massive aggregation and condensation of the lipid bilayers induced by the peptide. CAM interacts strongly with the negatively charged bilayer and induces extensive vesicle disruption, forming a condensed 'onion-like' multilamellar structure with repeat distance $d=2 \pi / q_{0} \sim 50.0 \AA$ as derived from the maximum of the observed peak and confirmed by SAXS (not shown). Fig. 3 shows a sketch of the proposed multilamellar structure of CAM-POPE/POPG mixture. We propose that this peptide exerts its antimicrobial action through extensive membrane disruption, leading to cell death.

In another study (Silva et al., 2013), the bilayer composed of dimyristoylphosphatidylcholine/dimyristoylphosphoglycerol (DMPC/DMPG = 3:1 $\mathrm{mol} / \mathrm{mol}$ ) mimicking the membrane of Candida albicans interacted with AMP of the lactoferrin family (LFchimera, nominal charge +12 ). Fig. 4 shows the SAXS pattern of two bicontinuous cubic phases detected due to disintegration of the membrane by pore-forming mechanism. These results illustrate variedness in the antimicrobial mechanism of AMPs acting on the lipid membrane. 


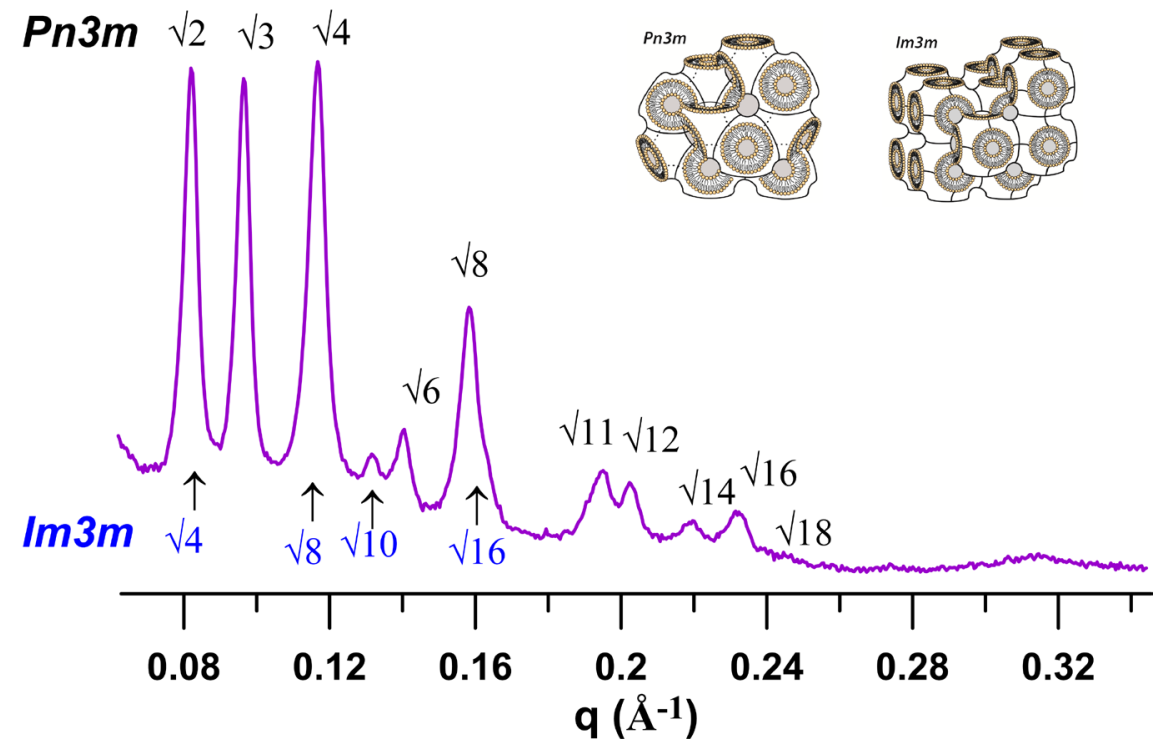

Figure 4. SAXS pattern of a mixture of LFchimera and DMPC/DMPG at $P: L=1: 21 \mathrm{~mol} / \mathrm{mol}\left(t=24^{\circ} \mathrm{C}\right)$. Peaks of detected cubic phases Pn3m (black) and Im3m (blue) are assigned to spacing $\sqrt{ }\left(h^{2}+k^{2}+l^{2}\right)$, where $h, k$ and l are Miller indices. Models of cubic phases of Pn 3 m and Im $3 m$ space group.

\section{LIPID BILAYERS IN DNA DELIVERY SYSTEM}

Lipoplexes are formed due to the electrostatic interaction between positively charged liposomes and DNA polyanion. Positively charged complexes show enhanced interaction with the negatively charged cytoplasmic membrane and, therefore, higher cellular uptake via endocytosis. However, for successful DNA delivery, lipoplexes must escape from the endosome inside the cell and DNA must be released from the complex in cytoplasm. High positive surface charge density of the lipoplex is crucial for a successful endosomal fusion. On the other hand, it can be an obstacle for DNA release from the complex. A way to overcome this problem is to use $\mathrm{pH}$-sensitive surfactants with $p K_{a}$ between 4.5 and 8 . Acidic $\mathrm{pH}$ inside of endosome secures lipoplexes' fusion with the endosomal membrane. In the cytoplasm, at neutral $\mathrm{pH}$, the surfactants are uncharged and DNA can be easily released from the lipoplex via dissociation.

We prepared $\mathrm{pH}$-sensitive liposomes composed of homologues of series of $\mathrm{N}, \mathrm{N}$-dimethyl-alkane-1-amine $N$-oxides ( $C n N O, n=8-18$, where $n$ is the number of carbon atoms in the alkyl substituent) (Devínsky et al., 1978) and neutral phospholipid dioleoylphosphatidylethanolamine (DOPE) at a molar ratio $\mathrm{C} n \mathrm{NO} / \mathrm{DOPE}=0.4$ and tested them for in vitro transfection activity. Several techniques (SAXS, UVVIS, zeta potential measurements, confocal microscopy) were applied to characterise the system in an effort to unravel the relationship between the transfection efficiency, structure and composition of the lipoplexes (for details, see Liskayová et al., 2019). Fig. 5 summarises the most important findings underlining the connection between structural changes (studied by SAXS) and transfection activity.
In acidic conditions, DNA-C8NO/DOPE at C8NO/DOPE $=0.4$ $\mathrm{mol} / \mathrm{mol}$ forms a condensed inverted hexagonal phase $\left(H_{\|}{ }^{\top}\right)$. In this structure, the DNA strands are inserted in hexagonally arranged tubules created by the surfactant/lipid mixture (Fig. 1B). Note that the complexes prepared with C8NO keep their structure in neutral/alkaline solutions also. CnNOs with longer hydrophobic alkyl substituent, $n \geq 10$, induce structural changes. SAXS revealed the coexistence of two phases, condensed lamellar $L_{a}^{C}$ phase and $H_{\| 1}{ }^{C}$ in the complexes DNA-CnNO/DOPE, $n \geq 10$. Commensurate lattice parameters, the repeat distance $d_{L C}$ of $L_{a}{ }^{C}$ phase and the lattice parameter of the $H_{\|}{ }^{C}$ phase $\left(d_{L C} \approx a_{H C}\right)$ indicate an epitaxial relationship, we abbreviate the structure as $L_{a}{ }^{C} \& H_{\| l}^{C}$. The connection through the common scattering plane facilitates the transition of the lipid between the two different structures. The structure changed in neutral/ slightly alkaline solutions. A lamellar phase $\left(L_{\alpha}\right)$ is a dominant structure in all studied mixtures. In addition, SAXS patterns of complexes DNA-CnNO/DOPE, $n=16$ and 18 , indicate the presence of a bicontinuous $\mathrm{Pn} 3 \mathrm{~m}$ cubic phase. The lattice parameter $a_{\mathrm{Q}}$ was found to be $a_{\mathrm{Q}} \sim 17-18 \mathrm{~nm}$ for detected cubic phases. Bicontinuous cubic phases $\left(Q_{\|}\right)$are formed by a pair of interpenetrating, but non-contacting aqueous channels separated by a single, continuous lipid bilayer. Even if the lattice parameter of Pn3m is rather big (17-18 nm), three-dimensional arrangement of lipid bilayer in the cubic network requires its high negative curvature that might bring difficulties to accommodate bulky DNA molecules. To summarise, the change of $\mathrm{pH}$ from acidic to neutral induces phase transition $L_{\alpha}{ }^{C} \& H_{\| 1}{ }^{C} \rightarrow Q_{\| 1}+L_{\alpha^{\prime}}$ which allows DNA release from the lipoplexes. 


\section{Lipoplexes DNA-CnNO/DOPE}
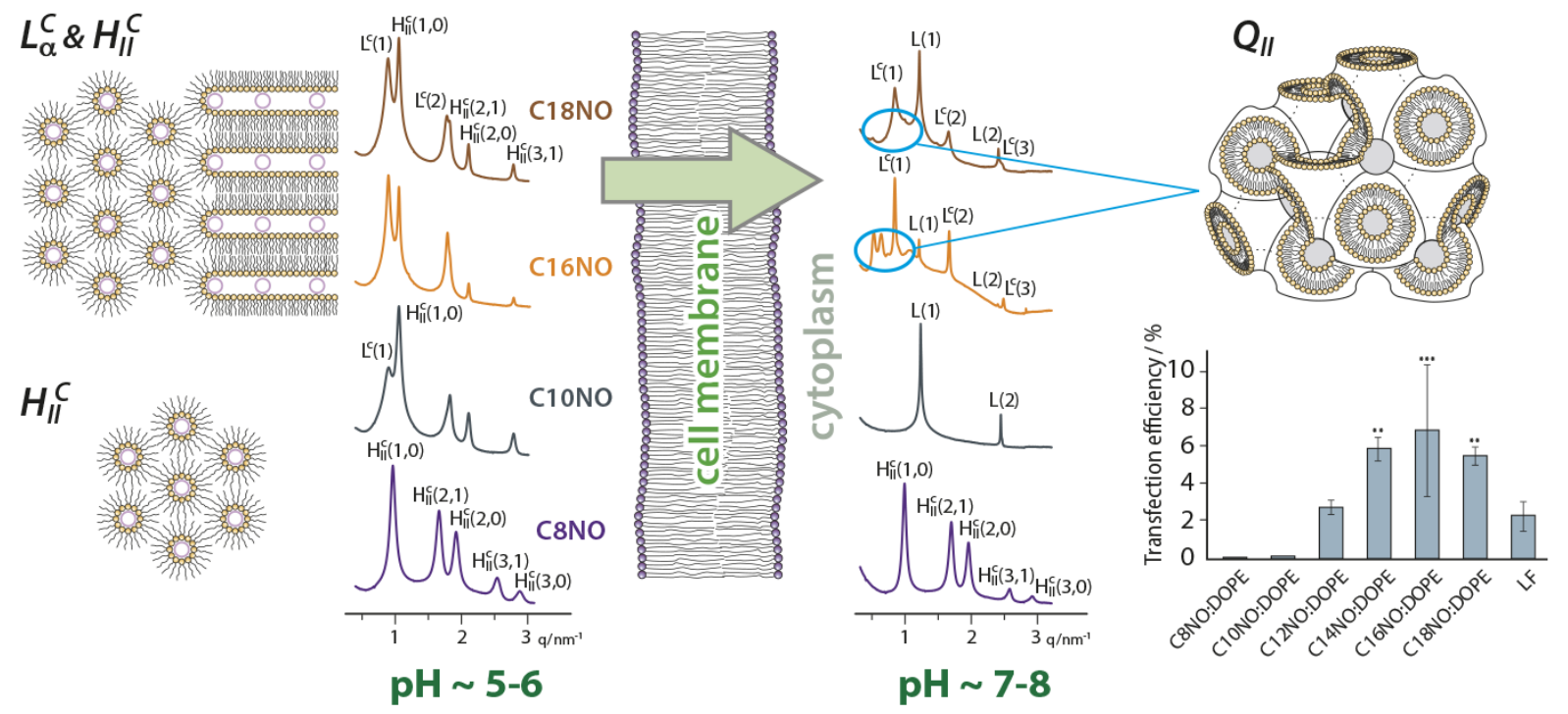

Figure 5. SAXS patterns of DNA-CnNO/DOPE complexes $(n=8,10,16$ and 18) prepared at CnNO/DOPE $=0.4 \mathrm{~mol} / \mathrm{mol}$ in aqueous solutions at acidic and neutral/slightly alkaline conditions, respectively. Transfection efficiency of CnNO/DOPE $(n=8-18)$ complexes tested on U2OS cells.

Transfection efficiency for plasmid DNA (pDNA, EGFP-N1) was tested on human bone osteosarcoma epithelial cells (U2OS line) and evaluated after 24 and 48 hours by flow cytometry. Commercially available Lipofectamine 2000 (LF) was used as a control. Transfection efficiency follows a quasi-parabolic dependence on the length of CnNO alkyl substituent $n$, with the maximum found at $n=16$. The complex of $\mathrm{C} 16 \mathrm{NO} /$ DOPE was found to be $\sim 3$ times more efficient than LF. Our findings support the hypothesis that the lipids of $Q_{||}$phase favour membrane pore formation resulting from fusion of the lipoplex cubic structure with endosomal membrane. Pores, in turn, allow for cytoplasmic gene delivery. Due to $\mathrm{pH}$ sensitivity of our lipoplexes, we can hypothesise synergy of two processes enhancing transfection: a massive release of DNA during $L_{\alpha}{ }^{\mathrm{C}} \& H_{\| 1}{ }^{C} \rightarrow Q_{\| 1}+L_{\alpha}$ phase transition and its easier internalisation in the cell supported by the propensity of $Q_{\| 1}$ for pore formation.

\section{LIPIDS AS A DRUG}

Pulmonary surfactant (PS) is a surface active film lining the alveoli of the lung (Fig. 6A). Its principal function is to lower the surface tension at the air/liquid interface, facilitate the exchange of gasses and stabilise alveoli during breathing. PS is composed of $\sim 90 \%$ lipids and $8 \%-10 \%$ of a few specific surfactant-associated proteins. Phospholipids predominate; saturated dipalmitoylphosphatidylcholine (DPPC) is the most abundant in PS of mammals, up to $\sim 50 \%$ by mass. Unsaturated phosphatidylcholines (PC) create $\sim 20 \%$ and a smaller fraction $(\sim 10 \%)$ of anionic species such as POPG.
A high content of DPPC plays a key function to reach the necessary, extremely low surface tension $(<1 \mathrm{mN} / \mathrm{m})$. Hydrophilic SP-A, SP-D and hydrophobic SP-B, SP-C are surfactant-associated specific proteins. SP-B is crucial for proper spreading of the lipid interfacial monolayer. The surface active function of PS is critically dependent on the existence of a multilayered film ( $100 \mathrm{~nm}$ in thickness) at the air-water interface. An inactivation, deficiency or an absence of PS results in respiratory distress syndrome (RDS) that can be lethal for premature babies. RDS is therapeutically treated by application of PS preparations obtained from animals (exogenous natural), like porcine Curosurf ${ }^{\oplus}$ or bovine Survanta ${ }^{\oplus}$.

The exogenous natural PS contains at least 50 different phospholipids and a small fraction of hydrophobic proteins ( 1-2 wt\%) (Calkovska et al., 2016). Morphologically, it is a mixture of various vesicles, from unilamellar through oligolamellar up to multilamellar.

We studied the effect of bacterial toxin, lipopolysaccharide (LPS), on the structure of clinically modified porcine pulmonary surfactant, mimicking pathological conditions. Consecutively, Polymyxin B (PxB), a cyclic amphiphatic antibiotic, was applied. Fig. 6B shows the SAXS patterns of modified porcine surfactant at $37^{\circ} \mathrm{C}$ when the mixture is in liquid crystalline state (fluid phase). Two peaks ( $L 1$ and $L 2$ ) belong to a lamellar phase with repeat distance $d \sim 8.35 \mathrm{~nm}$. LPS affects the multilamellar packing of PS. Longer incubation ( 2 2 hours) generates a large swelling up, which corresponds to a SAXS pattern of large unresolved peak of low intensity with a subtle shoulder at low $q \sim 0.5 \mathrm{~nm}^{-1}$. The pattern indicates 


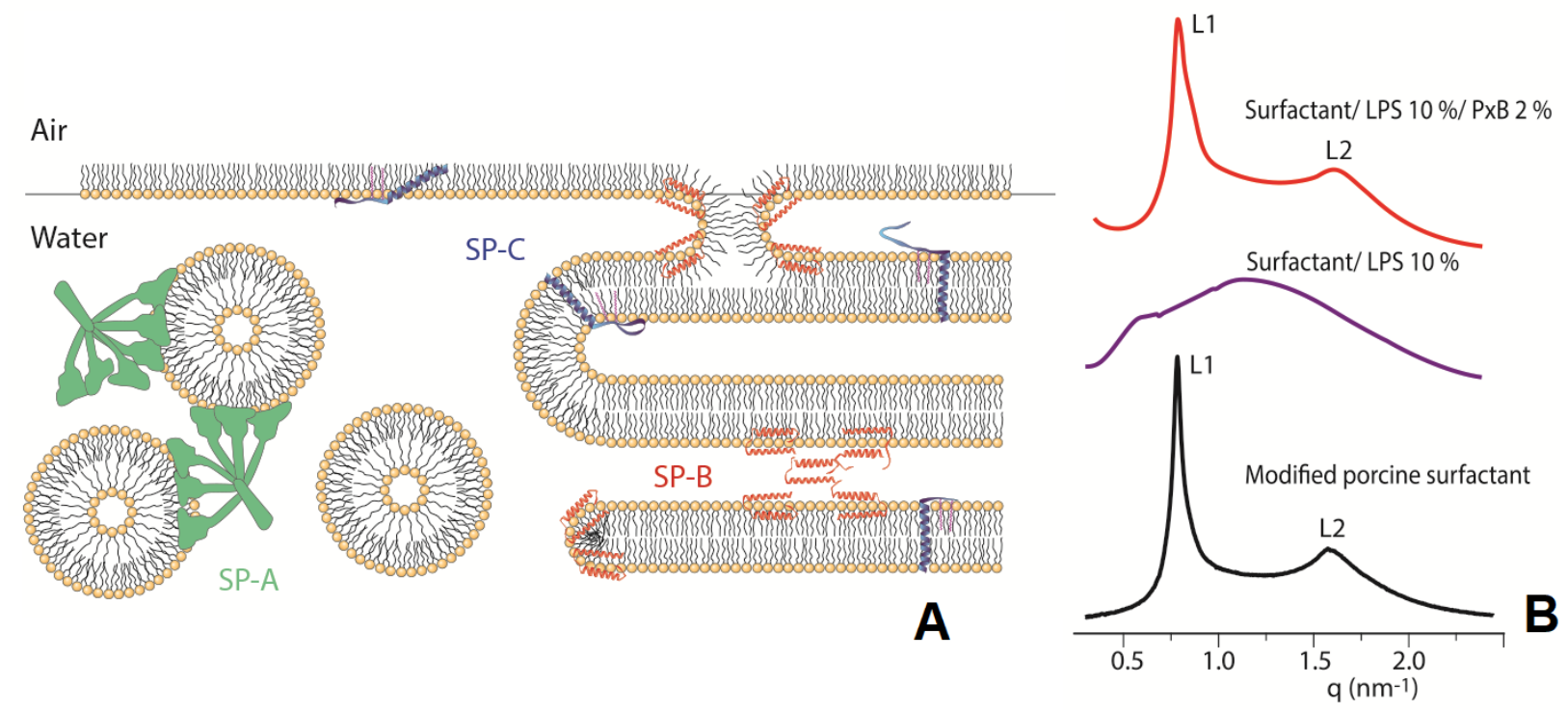

Figure 6. (A) Schematic representation of pulmonary surfactant; (B) SAXS pattern of modified porcine surfactant, surfactant incubated with $10 \%$ of LPS for 2 hours and surfactant/LPS $10 \%$ with addition of $2 \%$ of PXB $\left(\right.$ at $\left.37^{\circ} \mathrm{C}\right)$.

swelling of a lamellar phase up to periodicity $\sim 12-13 \mathrm{~nm}$. We attribute these structural changes to the PS surface charge unbalance due to LPS insertion. Consecutively, damaged surfactant/LPS $10 \%$ was incubated with peptide-based antibiotic PxB. We found that even a small dose of the cationic antibiotic reduces the repeat distance. PS/LPS incubated with $0.5 \%$ of $\mathrm{PxB}$ for $30 \mathrm{~min}$ reduced the repeat distance to $d \sim 8.6$ $\mathrm{nm}$ (not shown). Cationic molecule of PxB acts as an inhibitor of structural disarrangement induced by LPS and restores original lamellar packing. Structural changes were confirmed also by optical microscopy.

The study was focused on the mechanism underlying the structural changes in surface-reducing features (for details, see Kolomaznik et al., 2018). The function of 'infected' and consecutively 'treated' PS was tested with pulsating bubble surfactometer. The infected PS (surfactant/LPS 10\%) was not able to reach the necessary physiologically relevant low surface tension. Intriguingly, the minimal surface tension decreased alongside with structural recovery when applying PxB (Kolomaznik et al., 2018). The obtained results accurately reflect the situation with a native lung surfactant as confirmed by a recent in vivo study (Calkovska et al., 2021) and support the idea of PxB/Curosurf combined therapy in neonatal medicine.

\section{CONCLUDING REMARKS}

Amphiphilic molecules of lipids and surfactants self-assemble into structures of various morphologies ranging from micelles, lamellar and non-lamellar phases to microemulsions. Each of these morphologies possesses its own unique physical properties and offers the possibility for pharmaceutical applications. In the text above, we demonstrated just a few examples of 'usability' of phospholipid bilayers: as a model membrane mimicking the lipid bilayer of bacterial membrane in an effort to unravel the mechanisms of AMPs' antimicrobial activity, as a carrier designed for genetic material delivery, and finally, a lipid mixture extracted from animal's lungs that is clinically used in neonatal medicine. Findings presented here result from the collaboration between several institutions and have been published to the full extent in Kolomaznik et al. (2018), Liskayová et al. (2019), Silva et al. (2013) and Silva et al. (2018).

\section{ACKNOWLEDGEMENTS}

The author thanks all the co-authors in references (Kolomaznik et al., 2018; Liskayová et al., 2019; Silva et al., 2013, 2018) for their fruitful collaboration. SAXS experiments were performed at BL11-NCD-SWEET beamline at Alba Synchrotron, Barcelona with the collaboration of ALBA staff and at A2 beamline, Doris synchrotron in Hamburg. The research leading to these results has received funding from the JINR project 04-4-11422021/2025 and grants from VEGA 1/0223/20, APVV-17-0250 and SK-PT-18-0032. 


\section{References}

[1] Calkovska A, Haegerstrand-Björkman M, Curstedt T. Restoration of surfactant activity by polymyxin B in lipopolysaccharide potentiated injury of immature rabbit lungs. Scientific Reports $2021 ; 11: 22$

[2] Calkovska A, Linderholm B, Haegerstrand-Björkman M, Pelizzi N, Johansson J, Curstedt T. Phospholipid composition in synthetic surfactants is important for tidal volumes and alveolar stability in surfactant-treated preterm newborn rabbits. Neonatology 2016; 109: 177-185

[3] Devínsky F, Lacko I, Nagy A, Krasnec L'. Amine oxides. I. Synthesis, $1 \mathrm{H}$-n.m.r., and Infrared spectra of 4-alkylmorpholine-N-oxides. Chem. Pap. 1978; 32:106-115

[4] Israelachvili JN, Mitchell DJ, Ninham BW. Theory of self-assembly of hydrocarbon amphiphiles into micelles and bilayers. J. Chem. Soc. Faraday Trans 2, Mol. Chem. Phys. 1976; 72:1525-1568

[5] Kobayashi T, Tsukagoshi S, Sakurai K. Enhancement of the cancer chemotherapeutic effect of cytosine arabinoside entrapped in liposomes on mouse leukemia L-1210. Gann 1975; 66:719-720

[6] Kolomaznik M, Liskayova G, Kanjakova N, Hubcik L, Uhrikova D, Calkovska A. The perturbation of pulmonary surfactant by bacterial lipopolysaccharide and its reversal by Polymyxin B: function and structure. Int. J. Mol. Sci. 2018; 19: 1964

[7] Liskayová G, Hubčík L, Búcsi A, Fazekaš T, Martínez JC, Devínsky F, Pisárčik $M$, Hanulová $M$, Ritz $S$, Uhríková D. pH-sensitive $\mathrm{N}, \mathrm{N}$-dimethylalkane-1-amine $\mathrm{N}$-oxides in DNA delivery: from structure to transfection efficiency. Langmuir 2019; 35: 1338213395

[8] Silva T, Adao R, Nazmi K, Bolscher JGM, Funari SS, Uhríková D, Bastos M. Structural diversity and mode of action on lipid membranes of three lactoferrin candidacidal peptides. Biochim. Biophys. Acta 2013; 1828:1329-1339

[9] Silva T, Claro B, Silva BFB, Vale N, Gomes P, Gomes MS, Funari SS, Teixeira J, Uhríková $D$, Bastos $M$. Unravelling a mechanism of action for a cecropin -A-Mellitin hybrid antimicrobial peptide: the induced formation of multilamellar lipid stacks. Langmuir 2018; 34:2158-2170

[10] Teixeira V, Feio MJ, Bastos M. Role of lipids in the interaction of antimicrobial peptides with membranes. Prog. Lipid Res. 2012; 51:149-177. 